\title{
碳化铇在对硝基苯酚电还原过程中的电催化行为 *
}

\author{
马淳安黄烨童少平张维民 \\ (浙江工业大学应用化学系, 绿色化学合成技术国家重点实验室培育基地, 纳米科学与技术工程研究中心, 杭州 310032)
}

\begin{abstract}
摘要 以碳化铇(WC)粉末为电催化材料制成了碳化铇粉末微电极 (WC-PME). 采用循环伏安和线性扫描等方法 研究了酸性溶液中对硝基苯酚(PNP)在WC-PME上的电还原行为. 研究表明, 在相同测试条件下, PNP在WC-PME 上电还原的电位比 $\mathrm{Cu}-\mathrm{Hg}$ 微电极正得多; WC-PME 对氢具有较强的吸附能力, 有利于有机物的电还原反应. PNP 在 WC-PME 上和 Pt 微电极上的还原电位相近, 但在 WC-PME 上的峰电流比在 Pt 微电极上高 5 倍多, 这主 要与 $\mathrm{WC}$ 粉末的结构形貌有关.
\end{abstract}

关键词: 碳化铇, 粉末微电极, 对硝基苯酚, 电化学还原, 电催化 中图分类号： O646

早在 20 世纪 70 年代, 人们已对碳化铇(WC)的 催化性能做了大量的研究. 人们指出, $\mathrm{WC}$ 表面电子 层与铂类似, 在某些反应中具有类铂的催化活性 ${ }^{[-9]}$. 例如 WC 可用来代替 Pt 催化芳香族硝基化合物的 液相加氢 ${ }^{[-3]}$, 以及用于 $\mathrm{H}_{2} 、 \mathrm{CO}$ 反应的电催化剂和甲 醇等为原料的燃料电池中作为阳极氧化反应的催 化剂 ${ }^{[4-9]}$; 而且 WC 和铂催化剂的析氢过电位都较 小 ${ }^{[10-12]}$, 对氢有较强的吸附能力和电催化活性.

水溶液中析氢和有机物体系中的电催化还原反 应均需要对氢有较强吸附能力的电极材料, 电极上 氢吸附能力越强, 有机物电还原反应越易进行; 所以 选择具有氢吸附能力强、电催化活性高的电极材料, 是有机物电还原反应的关键问题之一. 据文献报道, WC 具有良好的析氢性能和氢离子化的电催化活 性 ${ }^{[1-122]}$, 但将 $\mathrm{WC}$ 应用于硝基化合物的电还原反应 的报道甚少 ${ }^{[13]}$. 芳香族硝基化合物的电还原反应是有 机电化学领域中一类重要的化学反应, 也是绿色化 学合成中的重要组成部分. 为此, 我们将纳米 WC 粉体制成粉末微电极, 采用循环伏安和线性扫描等 方法探讨 WC 催化剂在对硝基苯酚(PNP)电还原过 程中的电催化行为及其电还原性能.

\section{1 实验部分}

\section{$1.1 \mathrm{WC}$ 粉体的制备}

以偏铇酸铵为前躯体, $\mathrm{CO} / \mathrm{CO}_{2}$ 还原性气体为碳 源, 采用喷雾干燥-气固反应法制备具有介孔结构空 心球状的纳米WC 粉体 ${ }^{[1] !}$. 样品表面形貌用Philips XL30E型扫描电子显微镜(SEM)进行表征.

\section{2 碳化铇粉末微电极(WC-PME)的制备}

将直径为 $60 \mu \mathrm{m}$ 的铂丝一端与导线相连, 另一 端与玻璃管熔封在一起, 将封有铂丝的一端磨平抛 光制成铂微盘电极, 然后将 $\mathrm{Pt}$ 微电极放人沸腾的王 水中腐蚀, 控制腐蚀的时间得到深度为 $52 \mu \mathrm{m}$ 的空 穴; 通过充分挤压和研磨, 在空穴内填实 WC 粉末, 即制成 WC-PME ${ }^{[15]}$.

\section{3 电化学测试}

电化学测试用CHI660B型电化学工作站, 三电 极体系: 工作电极为WC-PME, 参比电极为饱和甘录 电极 (SCE), 对电极为大面积 Pt片. 在文中提到的电 位均相对于SCE, 峰电流已扣除背景电流. 作为对比 电极的 $\mathrm{Cu}-\mathrm{Hg}$ 微电极和 $\mathrm{Pt}$ 微电极的半径分别为 80 $\mu \mathrm{m}$ 和 $30 \mu \mathrm{m}$. 实验测试在 $(298 \pm 0.1) \mathrm{K}$ 下进行(明确 标明温度除外). 在测试之前电解液先鼓 $\mathrm{N}_{2} 30 \mathrm{~min}$, 以除去电解液中的溶解氧.

\section{2 结果与讨论}

\section{$2.1 \mathrm{WC}$ 粉体的表征}

图1为不同WC粉末的扫描电镜照片. 图1(a)为以

2004-11-23 收到初稿, 2005-01-04 收到修改稿. 联系人:马淳安(E-mail: science@ zjut. edu. cn; Tel: 0571-88320011).＊国家基础研究重 大项目前期研究专项基金(2003CCA01300)、国家自然科学基金(20476097, 20376074)和浙江省自然科学重点基金(ZD0024)资助项目 


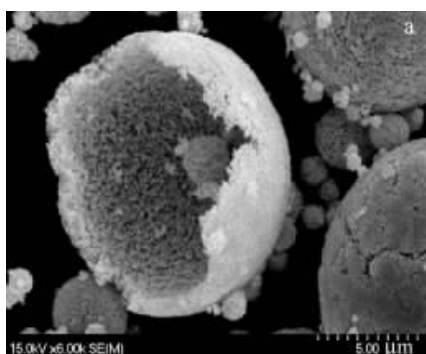

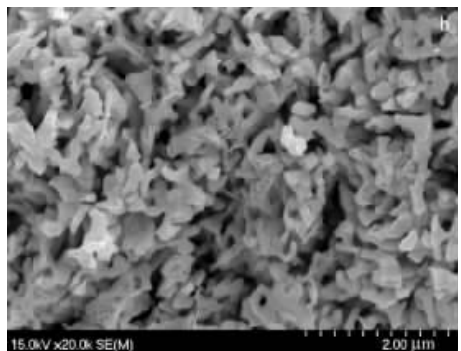

图 1 不同 WC 粉末的 SEM 图

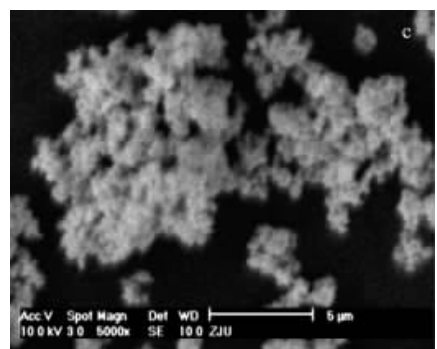

Fig.1 SEM images of different WC samples

Mean diameters of the samples (a), (b), and (c) are 90, 210, and $620 \mathrm{~nm}$, respectively

偏铇酸铵为前躯体, $\mathrm{CO} / \mathrm{CO}_{2}$ 还原性气体为碳源, 采 用喷雾干燥-气固反应法制备的介孔结构空心球状 $\mathrm{WC}$ 粉体的表面形貌图; 图 1(b)为以偏铇酸铵为前 躯体, $\mathrm{CO} / \mathrm{CO}_{2}$ 还原性气体为碳源, 采用气固反应法 制备的 WC 粉体的表面形貌图; 图 1(c)为株洲硬质 合金厂生产的 WC 粉体的表面形貌图. 从图中可观 察到, 图 1(a)中 WC 粉体的颗粒形状为空心球, 表面 具有介孔结构, 而且球体表面布满了凹凸不平的孔 隙, 由大小不一的短柱状小颗粒构成, 短柱状小颗粒 之间由形状各异、大小不等的孔隙构成, 微球表面介 孔的平均粒径为 $90 \mathrm{~nm}$; 图 1(b)和图 1(c)均未形成 微球, 平均粒径分别为 210 和 $620 \mathrm{~nm}$.

图 2 给出了三种不同 $\mathrm{WC}$ 粉末制成的微电极 在 PNP 体系中的线性扫描曲线. 从图中可以看出, 三条曲线中的第二个峰电位所对应的 PNP 电还原 反应极化峰电流分别为 $0.055,0.041$ 和 $0.019 \mathrm{~A} \cdot \mathrm{cm}^{-2}$, 说明这三种不同结构形貌的 WC 粉体的电催化活 性是不一样的. 其中采用喷雾干燥-气固反应法制备 的具有介孔结构空心球状的 WC 粉末所对应的峰

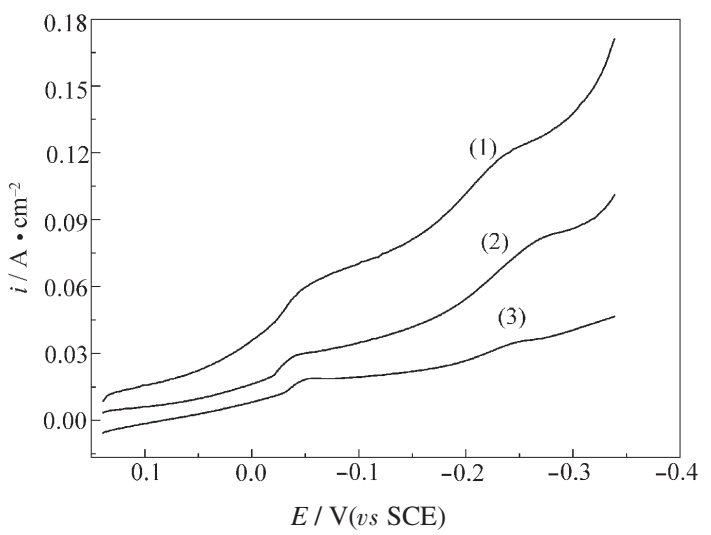

图 2 不同 WC 粉末微电极在 PNP 体系中的线性扫描曲线

Fig.2 Linear sweep voltammograms of $p$-nitrophenol (PNP) on different WC-PMEs

$c(\mathrm{PNP})=0.01 \mathrm{~mol} \cdot \mathrm{L}^{-1} ; c\left(\mathrm{H}_{2} \mathrm{SO}_{4}\right)=1.0 \mathrm{~mol} \cdot \mathrm{L}^{-1}$;

scan rate: $25 \mathrm{mV} \cdot \mathrm{s}^{-1}$. (1), (2) and (3) corresponding to the samples (a), (b) and (c) in Fig.1, respectively.
电流最大, 电催化活性最高. 由此可见, WC 粉末的 电催化性能与其制备方法和结构形貌相关.因此, 选 用电催化性能最佳、具有介孔结构空心球状的 WC 样品来制备 WC-PEM, 同时对其催化 PNP 电化学还 原性能进行探讨.

\subsection{PNP 在 WC-PME 上的循环伏安研究}

图 3 为 $\mathrm{PNP}$ 在 $\mathrm{WC}-\mathrm{PME}$ 上的 $\mathrm{CV}$ 图. 图中曲 线 $\mathrm{A}$ 显示的是空白体系, 即 $\mathrm{WC}-\mathrm{PME}$ 在 $1.0 \mathrm{~mol} \cdot \mathrm{L}^{-1}$ $\mathrm{H}_{2} \mathrm{SO}_{4}$ 中的循环伏安图, 从图中可见随着电位向负 扫描, 电流升高, 然后趋于平台, 这种行为归因于氢 的吸附. 峰(a)对应于 $\mathrm{H}^{+}$还原为 $\mathrm{H}_{\mathrm{ads}}$ 的反应. $-0.28 \mathrm{~V}$ 所对应的峰 (d) 为析氢峰. 而在反向扫描时出现的峰 (b)则对应于 $\mathrm{H}_{\mathrm{ads}}$ 的氧化. Armstrong 等 ${ }^{[16]}$ 在研究 1.0 $\mathrm{mol} \cdot \mathrm{L}^{-1} \mathrm{H}_{2} \mathrm{SO}_{4}$ 中 $\mathrm{WC}$ 对析氢反应的电催化行为时, 也得到了类似的结果. 在体系中加人 $0.01 \mathrm{~mol} \cdot \mathrm{L}^{-1}$ 的PNP (图3曲线B), 发现在发生 $\mathrm{H}^{+}$吸附反应的峰(a) 处电流开始增大, 并在 $-0.18 \mathrm{~V}$ 处出现一个新的峰 (c), 该峰为 PNP 的电还原峰.

\subsection{PNP 在WC-PME与 $\mathrm{Cu}-\mathrm{Hg}$ 微电极的线性扫描}

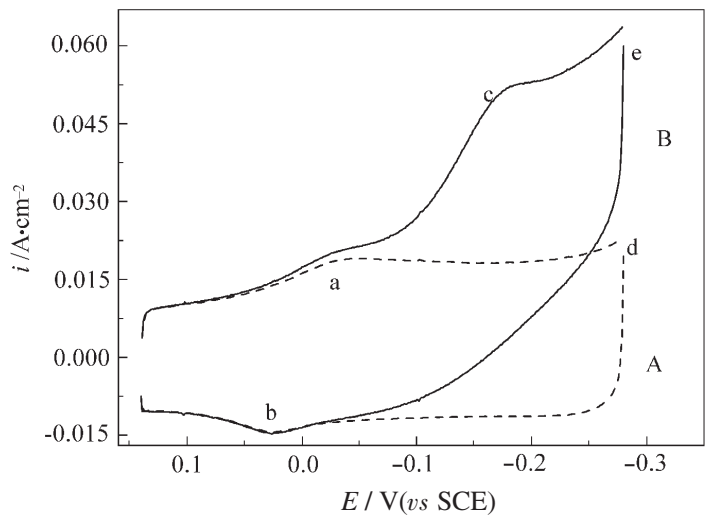

图 3 PNP 在 WC-PME 上的循环伏安图

Fig.3 Cyclic voltammograms of PNP on WC-PME

scan rate: $25 \mathrm{mV} \cdot \mathrm{s}^{-1}$. A) $\left.1.0 \mathrm{~mol} \cdot \mathrm{L}^{-1} \mathrm{H}_{2} \mathrm{SO}_{4} ; \mathrm{B}\right)=0.01 \mathrm{~mol} \cdot \mathrm{L}^{-1}$ $\mathrm{PNP}+1.0 \mathrm{~mol} \cdot \mathrm{L}^{-1} \mathrm{H}_{2} \mathrm{SO}_{4}$. a) the reduction of $\mathrm{H}^{+}$and Hads; b)the desorption of Hads; c)the reduction of $p$-nitrophenol; d)hydrogen evolution 


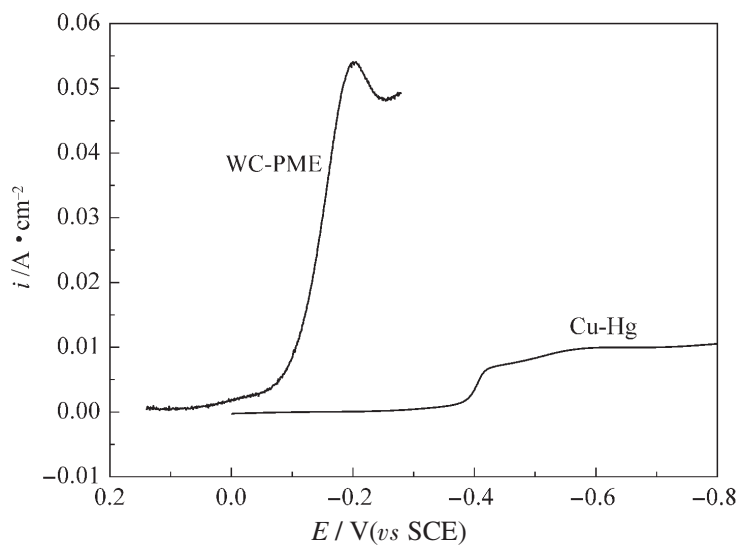

图 4 PNP 的线性扫描图

Fig.4 Linear sweep voltammograms of PNP

Background current has been subtracted. Scan rate: $25 \mathrm{mV} \cdot \mathrm{s}^{-1}$; $c(\mathrm{PNP})=0.01 \mathrm{~mol} \cdot \mathrm{L}^{-1} ; c\left(\mathrm{H}_{2} \mathrm{SO}_{4}\right)=1.0 \mathrm{~mol} \cdot \mathrm{L}^{-1}$

图4是PNP在WC-PME和 $\mathrm{Cu}-\mathrm{Hg}$ 微电极上的线性 扫描图. 从图可见, WC-PME和 $\mathrm{Cu}-\mathrm{Hg}$ 微电极在PNP 电还原体系中的波脚电位分别为 $-0.05 \mathrm{~V}$ 和 $-0.38 \mathrm{~V}$ 左右, PNP在WC-PME上的还原电位比在 $\mathrm{Cu}-\mathrm{Hg}$ 微电 极将近正 $0.33 \mathrm{~V}$, 且PNP在 WC-PME上电还原的峰电 流密度比 $\mathrm{Cu}-\mathrm{Hg}$ 微电极高3倍多.

WC 具有良好的析氢性能 ${ }^{10-12]}$, 而 $\mathrm{Cu}-\mathrm{Hg}$ 电极的 析氢过电位较高. 从 PNP 在两电极上的电还原电位 可以看出, WC 粉末对氢有较强的吸附能力, 有利于 有机物的电还原反应.

\subsection{PNP 在 WC-PME 与 Pt 微电极的线性扫描}

图5显示的是PNP在WC-PME和Pt 微电极上的 线性扫描图. 从图中可以看出, PNP在WC-PME和Pt 微电极上电还原的峰电位分别为 $-0.205 \mathrm{~V}$ 和 -0.215 $\mathrm{V}$, 非常接近, 同时在PNP电还原过程中都具有一定 的电催化活性. 但WC-PME和Pt 微电极在PNP电还 原反应时的峰电流却相差很大, 分别为 0.055 和 0.008

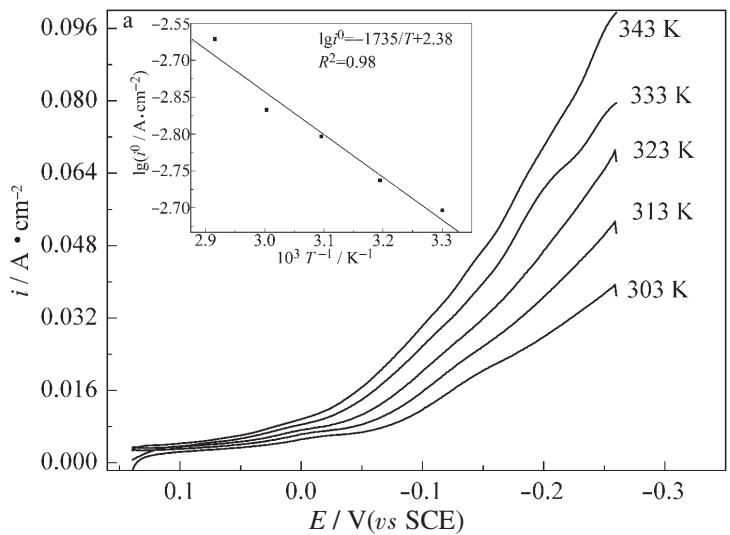

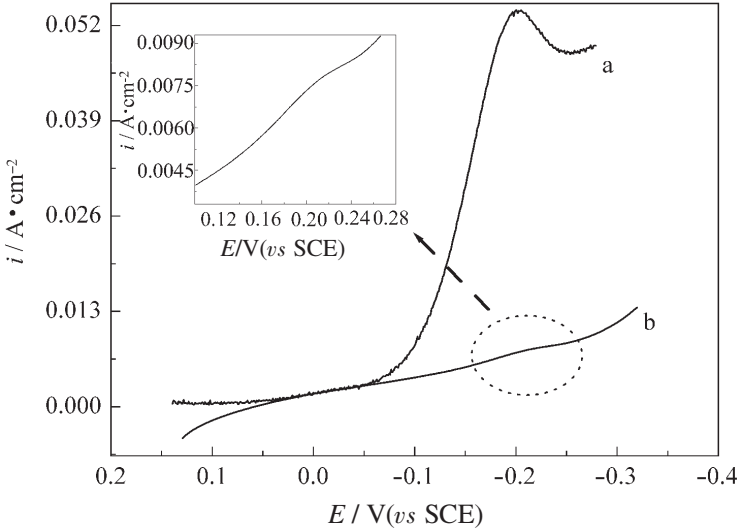

图 5 PNP 线性扫描图

Fig.5 Linear sweep voltammograms of PNP

Background current has been subtracted. (a) WC-PME;

(b) Pt microelectrode; scan rate: $25 \mathrm{mV} \cdot \mathrm{s}^{-1}$;

$c(\mathrm{PNP})=0.01 \mathrm{~mol} \cdot \mathrm{L}^{-1} ; c\left(\mathrm{H}_{2} \mathrm{SO}_{4}\right)=1.0 \mathrm{~mol} \cdot \mathrm{L}^{-1}$

$\mathrm{A} \cdot \mathrm{cm}^{-2}, \mathrm{WC}-\mathrm{PME}$ 的峰电流比Pt微电极高 5 倍多, 由 此可见, WC粉末对PNP的电催化活性大于Pt电极.

为了深人探讨 $\mathrm{WC}$ 与 $\mathrm{Pt}$ 微电极电催化活性之间 的差异, 究竟是源于几何因素还是能量因素 ${ }^{[17]}$, 需要 验证WC-PME 和Pt 微电极在反应过程中的表观活 化能. 图6给出了WC-PME和 Pt微电极在不同温度 下的准稳态极化曲线.依据图6所测定的稳态极化曲 线, 可求出交换电流密度 $i^{0}$, 然后以 $\lg i^{0}$ 与 $1 / T$ 作图 (见图6插图), 按照(1)式

$$
\lg i^{0}=\lg (F \cdot K \cdot c)-E_{\mathrm{a}} / 2.3 R T
$$

可分别求得 WC-PME 和 Pt 微电极在 PNP 电还原 过程中的表观活化能分别为 $(33.21 \pm 0.66) \mathrm{kJ} \cdot \mathrm{mol}^{-1}$ 和 $(22.34 \pm 0.22) \mathrm{kJ} \cdot \mathrm{mol}^{-1}$. 式中 $K$ 为常数, $F$ 为法拉第常 数, $c$ 为反应物浓度, $R$ 为气体常数, $T$ 为反应温度. 从计算所得的表观活化能可以看出, 也就是从能量 因素来看, WC粉末在PNP电还原体系中的电催化性

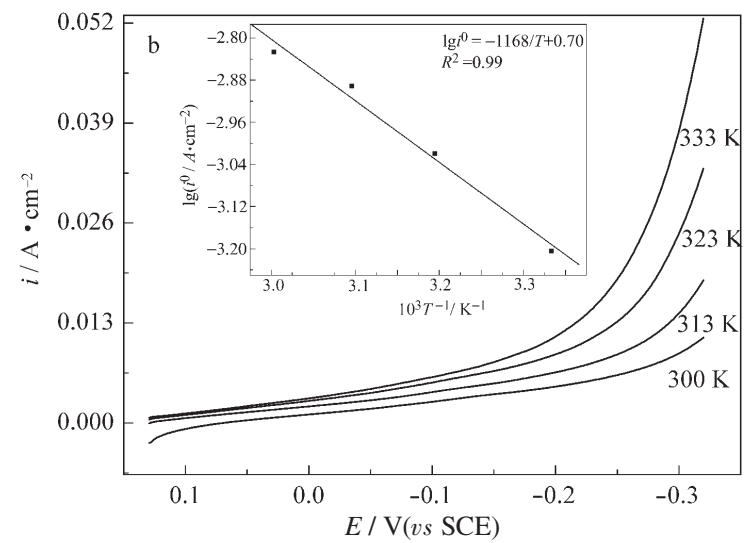

图 $6 P N P$ 在不同温度下的稳态极化曲线及其 $\lg i^{0}-1 / T$

Fig.6 Steady state polarization curves of PNP at various temperatures and its $\lg i^{0}-1 / T$

(a) WC-PME; (b) Pt microelectrode; $c(\mathrm{PNP})=0.01 \mathrm{~mol} \cdot \mathrm{L}^{-1} ; c\left(\mathrm{H}_{2} \mathrm{SO}_{4}\right)=1.0 \mathrm{~mol} \cdot \mathrm{L}^{-1}$. 
能应该不如Pt微电极, 但在实际体系中WC-PME电 还原的峰电流值却远高于Pt微电极, 出现这一现象 显然无法用能量因素来解释. 影响电极电催化性能 的主要因素中, 除了能量因素以外, 还有电极的几何 因素, 也就是催化剂的表面状况. 本研究中所用的 $\mathrm{WC}$ 粉体的结构形貌为空心球, 其表面具有介孔结 构, 可以认为 $\mathrm{WC}$ 粉末微电极比Pt微电极更优越的电 催化性能, 可归因于WC粉末的介孔形貌的表面结构.

\section{3 结 论}

(1)采用三种不同结构形貌的WC粉末, 制成了 碳化铇粉末微电极, 通过线性扫描法对各自的电催 化性能进行了评价, 结果表明, 具有介孔结构空心球 状的WC粉末微电极电催化活性最佳.

(2)通过循环伏安和线性扫描等方法研究了 PNP 在WC-PME上的电还原性能. 研究表明, PNP在WCPME上出现明显的电还原峰, 具有良好的电催化活 性; 在相同测试条件下, WC-PME在PNP电还原过程 中的性能比 $\mathrm{Cu}-\mathrm{Hg}$ 微电极优良, 对氢具有较强的吸 附能力, 有利于有机物的电还原反应.

(3)研究表明WC-PME和Pt微电极在PNP体系中 的还原电位相近, 但WC-PME的峰电流比Pt微电极 高5倍多. 碳化铇粉末微电极良好的电催化性能主要 与结构形貌有关.

\section{References}

1 Chen, J. G. Chem. Rev., 1996, 96 : 1477

2 Delannoy, L.; Giraudon, J. M.; Granger, P. J. Catal., 2002, 206 : 358

3 Santos, J. B. O.; Valenca, G. P.; Rodrigues, J. A. J. J. Catal., 2002, 210: 1

4 Böhm, H. Electrochim. Acta, 1970, 15: 1273

5 Fleischmann, R.;Böhm, H. Electrochim. Acta, 1977, 22 : 1123

6 Palanker, V. S. Electrochim. Acta, 1975, 20:51

7 Hwu, H. H.; Polizzotti, B. D.; Chen, J. G. J. Phys. Chem. B, 2001, 105: 10045

8 Ma, C. A.; Gan, Y. P.; Chu, Y. Q.; Huang, H.; Chen, D. H.; Zhou, B. X. Trans. Nonferrous Met. Soc. China, 2004, 14:11

9 Ma, C. A.; Zhang, W. K.; Chen, D. H.; Zhou, B. X. Trans. Nonferrous Met. Soc. China, 2002, 12:1015

10 Sokolsky, D. V.; Palanker, V. Sh.; Baybatyrov, E. N. Electrochim. Acta, 1975, 20:71

11 Armstrong, R. D.; Bell, M. F. Electrochim. Acta, 1978, 23:1111

12 Xiao, X. F.; Liu, R. F.; Zhu, Z. S. Acta Phys.-Chim. Sin., 1999, 15:742 [肖秀峰, 刘榕芳, 朱则善. 物理化学学报(Wuli Huaxue Хиеваo), 1999, 15: 742]

13 Horányi, G.; Vértes, G. J. Chem. Soc. Perkin Trans. II, 1975: 827

14 Ma, C. A.; Li, G. H.; Zhang, W. M. A kind of tungsten carbide catalyst and its preparation method and special carbonizer. China Patent, 200410067120. X. 2004 [马淳安, 李国华, 张维民.一种 碳化铇催化剂及其制备方法和专用碳化炉. 中国发明专利, 200410067120. X. 2004]

15 Cachet-Vicier, C.; Vicier, V.; Cha, C. S. Electrochim. Acta, 2001, 47:181

16 Armstrong, R. D.; Bell, M. F. Electrochim. Acta, 1978, 23:1111

17 Qin, Q. X.; Zhu, L. Z.; Liu, S. L.; Guo, H. T. Acta Phys. -Chim. Sin., 1994, 10:892 [覃奇贤, 朱龙章, 刘淑兰, 郭鹤桐. 物理化 学学报(Wuli Hиахие Хиebao), 1994, 10 : 892]

\title{
The Catalytic Behavior of Tungsten Carbide for the Electroreduction of $p$-nitrophenol *
}

\author{
MA, Chun-An HUANG, Ye TONG, Shao-Ping ZHANG, Wei-Min \\ (Department of Applied Chemistry, State Key Laboratory Breeding Base of Green Chemistry-Synthesis Technology, Research \\ Center of Nano Science \& Technology, Zhejiang University of Technology, Hangzhou 310032)
}

\begin{abstract}
Tungsten carbide powder microelectrode (WC-PME) was prepared from tungsten carbide (WC) powders to be used as an electrocatalyst. Electrochemical reduction behaviour of $p$-nitrophenol (PNP) on the WC-PME was investigated using cyclic voltammetry $(\mathrm{CV})$ and linear sweep voltammetry. It was found that reduction potential of PNP on the WC-PME was more positive than that on the $\mathrm{Cu}-\mathrm{Hg}$ microelectrode and that WC had higher catalytic activity in the electroredution of PNP than $\mathrm{Cu}-\mathrm{Hg}$. Although the reduction potentials of PNP on the WC-PME and the Pt microelectrode were close to each other, the reduction current of PNP on the WC-PME was about 5 times as large as that on the Pt microelectrode. This result was mainly attributed to the surface morphological structure of WC.
\end{abstract}

Keywords: Tungsten carbide, Powder microelectrode, $p$-nitrophenol, Electroreduction, Electrocatalyst

Received: November 23, 2004; Revised: January 4, 2005. Correspondent: MA, Chun-An (E-mail: science@zjut. edu. cn; Tel: 057188320011). * The Project Supported by National Basic Research Priorities Program (2003CCA01300), NSFC(20476097, 20276069), and NSF of Zhejiang Province (ZD0024) 